\begin{tabular}{|c|l|}
\hline Title & $\begin{array}{l}\text { SOME APPLICA TIONS OF THE METHOD OF TRA NSFER-MA TRIX TO THE VIBRA TION OF LATTICES } \\
\text { WITH FREE BOUNDARIES }\end{array}$ \\
\hline Author(s) & A SAHI, Takashi \\
\hline Citation & JOURNAL OF THE RESEARCH INSTITUTE FOR CA TALYYSIS HOKKA IDO UNIVERSITY, 11(3), 133-163 \\
\hline Issue Date & 196402 \\
\hline Doc URL & http://hdl.handle.net/2115/24768 \\
\hline Type & bulletin (article) \\
\hline File Information & 11(3)_P133-163.pdf \\
\hline
\end{tabular}

Instructions for use 


\title{
SOME APPLICATIONS OF THE METHOD OF TRANSFER-MATRIX TO THE VIBRATION OF LATTICES WITH FREE BOUNDARIES*
}

\author{
By \\ Takashi AsAHi** \\ (Received September 30, 1963)
}

\begin{abstract}
The vibration of lattices of one, two and three dimensions with free boundaries is studied by the method of transfer-matrix, making use of an artifice, the "method of image". The effects of isotopic impurity atoms in the inside of the lattice or on one of its surfaces are investigated in detail. Main results obtained are as follows: (1) A one-dimensional lattice with an isotopic impurity at one of its ends has a localized mode if the mass of the impurity is smaller than half of the mass of host atoms. (2) A two-dimensional lattice with one light isotopic impurity on one of its edges may have a localized mode. For the case in which the impurity is at the center of an edge or at a corner, an analytical expression for the critical value of the impurity mass for the appearance of the localized mode is obtained. (3) If all the atoms composing an edge of two-dimensional lattice are light isotopes with the same mass, there may appear surface modes, the frequencies of which may lie in the frequency band of unperturbed spectrum. (4) The dispersion relation for the eigenfrequencies of the lattice containing an impurity at an arbitrary lattice site is obtained.

Some discussions are given finally on the relation between our method and the conventional method of GREEN's function.
\end{abstract}

\section{Introduction}

Recently it was shown by the author ${ }^{1)}$ that the method of transfer-matrix, which had been devised by HORI and $\mathrm{ASAHI}^{2)}$ to treat the problems of the vibration of linear lattices, is applicable to the lattices with any dimension. The application to higher-dimensional lattices have hitherto been confined, however, exclusively to the case of cyclic boundary conditions. In this paper the lattices with free boundaries are treated.

Linear lattices with free ends have been treated by MATsudA ${ }^{3)}$. In $\S 2$ of this paper it is shown that the free-end condition can be taken into account

*) A part of this work was presented at the International Conference on Lattice Dynamics held at Copenhagen in August, 1963.

**) Department of Physics, Hokkaido University, Sapporo. 
more simply. In $\$ 3$ an one-dimensional lattice with an isotopic impurity atom at one of its end is considered. The condition for the appearance of the localized mode is examined and found to be in accordance with the result of MATsudA.

In $\S 4$, a two-dimensional regular monatomic lattice with free edges is considered by the generalized method of transfer-matrix. In this case one must introduce an artifice, the "method of image", in order to take the free edges into account. Once this is done, however, the calculation is straightforward. In $\S 5$, two-dimensional lattices with isotopic impurity atoms on one of the free edges are considered, and the following results are obtained:

(1) A two-dimensional lattice with one light isotopic impurity on one of the edges may have a localized vibrational mode. For the case in which the impurity lies at one of the corners or at the center of the edge, the expression for the critical value of mass of the impurity for the appearance of the localized mode is obtained analytically. From this it is seen that the localized mode appears less easily for the former than for the latter and for the one-dimensional lattice with an impurity at one of its ends.

(2) If all the atoms on one of the edges of the lattice are light isotopes, there appear surface modes which damp towards the inside of the lattice. The frequencies corresponding to these surface modes may lie both inside and outside the band.

The treatments for two-dimensional lattices are easily generalized to threedimensional ones. In $\S 6$, the calculations similar to that in $\$ 4$ are performed for a three-dimensional regular monatomic lattice with free surfaces. In $\$ 7$, a three-dimensional lattice with an isotopic impurity on one of the surfaces is considered. The results similar to those for two-dimensional lattices are obtained.

In $\S 8$, it is shown that the results which have been obtained by the usual GREEN's function method can be got as special cases of those which are deduced by the transfer-matrix method.

Throughout the paper, it is assumed that there are nearest-neighbor interactions only. It should be remarked that this assumption is essential for the validity of the "method of image".

\section{One-Dimensional Regular Monatomic Lattices with Free Ends}

At first consider an one-dimensional lattice with free ends composed of $N$ equally spaced atoms of the same kind. Let the mass of atom be $M$, and the nearest-neighbor force-constant $K$. Assuming the time factor $\exp (i \omega t)$, we have the equation of motion for the displacement $u(l)$ of the atom at the lattice point $l$ : 
Some Applications of the Method of Transfer-Matrix to the Vibration of Lattices

$$
-M \omega^{2} u(l)=K\left[\{u(l-1)-u(l)\}\left(1-\delta_{l, 2}\right)+\{u(l+1)-u(l)\}\left(1-\delta_{l, N}\right)\right],
$$

where the Kronecker deltas in Eq. (2.1) are introduced in order to take into account the free ends $l=1$ and $N$.

Eq. (2.1) is equivalent to the homogeneous equation

$$
-M \omega^{2} u(l)=K[u(l-1)-2 u(l)+u(l+1)],
$$

or

$$
u(l+1)=\left(2-M \omega^{2} / K\right) u(l)-u(l-1), \quad 1 \leqq l \leqq N,
$$

with boundary conditions

$$
u(0)=u(1) \text { and } u(N+1)=u(N) .
$$

Introducing the state vector $x(l)$ and the transfer matrix $T$ by

$$
x(l) \equiv\left(\begin{array}{l}
u(l) \\
u(l-1)
\end{array}\right)
$$

and

$$
T \equiv\left(\begin{array}{rr}
\gamma & -1 \\
1 & 0
\end{array}\right),
$$

where

$$
\gamma \equiv 2-M \omega^{2} / K
$$

Eqs. (2.2b) and (2.3) can be written in vector-matrix forms:

$$
\begin{aligned}
& x(l+1)=T x(l), \quad 1 \leqq l \leqq N, \\
& x(1)=\left(\begin{array}{l}
1 \\
1
\end{array}\right) u(1), \\
& x(N+1)=\left(\begin{array}{l}
1 \\
1
\end{array}\right) u(N) .
\end{aligned}
$$

The transfer matrix $T$ can be transformed into the diagonal form :

$$
T=P A P^{-1},
$$

where

$$
A \equiv\left(\begin{array}{ll}
\lambda_{1} & 0 \\
0 & \lambda_{2}
\end{array}\right),
$$




$$
\begin{aligned}
& P \equiv\left(\begin{array}{ll}
\lambda_{1} & \lambda_{2} \\
1 & 1
\end{array}\right), \\
& P^{-1} \equiv \frac{1}{\lambda_{1}-\lambda_{2}}\left(\begin{array}{rr}
1 & -\lambda_{2} \\
-1 & \lambda_{1}
\end{array}\right),
\end{aligned}
$$

and

$$
\lambda_{1} \equiv\left[\gamma \pm\left(\gamma^{2}-4\right)^{\frac{1}{2}}\right] / 2
$$

Putting $M \omega^{2}=4 K \sin ^{2} \theta$, we obtain

$$
\begin{aligned}
& \gamma=2 \cos 2 \theta, \\
& \lambda_{1}=\exp ( \pm i 2 \theta),
\end{aligned}
$$

and

$$
T^{\iota}=\left(\begin{array}{ll}
S(l+1) & -S(l) \\
S(l) & -S(l-1)
\end{array}\right)
$$

where

$$
S(l) \equiv \sin 2 l \theta / \sin 2 \theta .
$$

From Eq. (2.5a) we get

$$
x(N+1)=T^{v} x(1) .
$$

Combined with Eqs. $(2.5 \mathrm{~b}, \mathrm{c})$ and $(2.8 \mathrm{a})$, this gives

$$
\begin{aligned}
\left(\begin{array}{l}
1 \\
1
\end{array}\right) u(N) & =T^{N}\left(\begin{array}{l}
1 \\
1
\end{array}\right) u(1) \\
& =\left(\begin{array}{l}
S(N+1)-S(N) \\
S(N)-S(N-1)
\end{array}\right) u(1),
\end{aligned}
$$

and consequently

$$
A u(1)=0 \text {, }
$$

where

$$
A \equiv S(N+1)-2 S(N)+S(N-1)
$$

A non-trivial solution of Eq. (2.10a) exists if and only if

$$
A=0 \text {. }
$$

This condition leads to the equation

$$
\tan \theta \sin 2 N \theta=0 .
$$

From Eq. (2.12) we find

$$
\theta=\kappa \pi / 2 N, \quad \kappa=0,1,2, \cdots, N-1 .
$$


Substituting Eq. (2.13) in Eq. (2.7a) we obtain the well known eigenfrequencies:

$$
M \omega_{x}^{2}=4 K \sin ^{2} \varphi_{x},
$$

where

$$
\varphi_{\kappa}=\kappa \pi / 2 N, \quad \kappa=0,1 . \cdots, N-1 .
$$

From Eqs. (2.4a), (2.5a) and (2.8a), it follows that

$$
u(l)=[S(l)-S(l-1)] u(1) .
$$

Substituting Eq. (2.8b) and (2.14) into Eq. (2.15) we obtain the wave form of the normal vibration corresponding to the eigenfrequency $\omega_{\kappa}$ :

$$
u(l)=\cos \left\{(2 l-1) \varphi_{\kappa}\right\} \sec \varphi_{\kappa} u(1) .
$$

\section{One-Dimensional Monatomic Lattice with an Isotopic Impurity Atom at its End Point}

We consider next the case in which the $N$ th atom in the regular monatomic lattice treated in $\S 2$ is replaced by an isotopic impurity atom with mass $M^{\prime}$. When we write the equation of motion in the form of a vector-matrix equation as before, only one transfer matrix $T^{\prime}$ connecting $x(N)$ with $x(N+1)$ comes out to be different from that of the regular lattice $T$ :

$$
x(N+1)=T^{\prime} x(N),
$$

where

$$
T^{\prime} \equiv\left(\begin{array}{rr}
\gamma^{\prime} & -1 \\
1 & 0
\end{array}\right)
$$

and

$$
\gamma^{\prime} \equiv 2-M^{\prime} \omega^{2} / K
$$

Defining the parameters

$$
Q \equiv\left(M^{\prime}-M\right) / M
$$

and

$$
\delta \equiv Q M \omega^{2} / K
$$

we obtain

$$
T^{\prime}=T-\left(\begin{array}{ll}
\delta & 0 \\
0 & 0
\end{array}\right) .
$$

In the same way as in $\$ 2$, we get the equations

$$
x(N+1)=T^{\prime} T^{N-1} x(1)
$$

and 


$$
\begin{aligned}
\left(\begin{array}{l}
1 \\
1
\end{array}\right) u(N) & =T^{\prime} T^{N-1}\left(\begin{array}{l}
1 \\
1
\end{array}\right) u(1) \\
& =\left[\left(\begin{array}{l}
S(N+1)-S(N) \\
S(N)-S(N-1)
\end{array}\right)-\delta\left(\begin{array}{c}
S(N)-S(N-1) \\
0
\end{array}\right)\right] u(1) .
\end{aligned}
$$

The condition for the existence of a non-trivial solution is

$$
A-\delta B=0 \text {, }
$$

where

$$
A \equiv S(N+1)-2 S(N)+S(N-1)
$$

and

$$
B \equiv S(N)-S(N-1)
$$

This leads to the equation:

$$
\tan \theta \sin 2 N \theta\left[1+\frac{1}{2} \delta(1+\cot \theta \cot 2 N \theta)\right]=0,
$$

or

$$
\sin \theta \cos (2 N-1) \theta[\tan (2 N-1) \theta+(1+2 Q) \tan \theta]=0 .
$$

Then we have

$$
\tan (2 N-1) \theta+(1+2 Q) \tan \theta=0,
$$

which coincides with the result obtained by MATSUDA ${ }^{3)}$. From this it will be seen that the effect of the impurity is to decrease or increase the eigenfrequencies according as $Q>0$ or $Q<0$. The values of perturbed eigenfrequencies cannot, however, exceed those of the neighboring unperturbed frequencies. Moreover, the maximum eigenfrequency runs out from the frequency band if $Q<-\frac{1}{2}$.

The wave forms of the normal modes corresponding to the eigenfrequencies in the band are sinusoidal also in the present case. On the contrary, the normal mode corresponding to the extra-band eigenfrequency decays exponentially as the distance from the impurity increases. We shall call this mode "damping mode".

In order to evaluate the extra-band frequency we have to put

$$
\theta=\pi / 2+i \varepsilon
$$

where

$$
\varepsilon \gg 1 / N
$$

This restriction is necessary for the spatial localization of the damping mode.

Substituting Eq. (3.7a) into Eq. (3.5), we get 


$$
1+2 Q \cosh \varepsilon(\cosh \varepsilon-\sinh \varepsilon) \cong 0,
$$

where we used an approximation:

$$
\operatorname{coth} 2 N \varepsilon \cong 1 \text {. }
$$

From Eq. (3.7b) we obtain the relation between $Q$ and $\varepsilon$ :

$$
Q=-(1+\exp (-2 \varepsilon))^{-1} \text {. }
$$

This shows that there is a critical value of $Q$ for the appearance of the damping mode:

$$
Q_{r}=-\frac{1}{2} \text {. }
$$

The wave form of the damping mode comes out to be

$$
\begin{aligned}
u(l) & =\cos \{(2 l-1) \theta\} \sec \theta u(1) \\
& =(-1)^{l-1} \sinh \{(2 l-1) \varepsilon\} \operatorname{cosech} \varepsilon \cdot u(1) \\
& =(-1)^{N-l} \sinh \{(2 l-1) \varepsilon\} \operatorname{cosech}\{(2 N-1) \varepsilon\} u(N) \\
& \cong(-1)^{N-l} \exp \{-2(N-l) \varepsilon\} u(N),
\end{aligned}
$$

where

$$
\exp (-2 N \varepsilon) \cong 0
$$

\section{Two-Dimensional Regular Monatomic Lattices with Free Edges}

In a previous work"), we generalized the transfer-matrix method to higher dimensional lattices with the cyclic boundary condition. In this section we shall apply the generalized method to a two-dimensional regular monatomic lattice with free edges. In the same way as in $\$ 2$, the effect of these free edges can be replaced by suitable boundary conditions. Assuming the nearest-neighbor interaction, the equation of motion for one of the components of atomic displacement $u(l, m)$ at a lattice point $(l, m)$ can be written in the form :

$$
\begin{aligned}
&-M \omega^{2} u(l, m)=K_{1}\left[\{u(l-1, m)-u(l, m)\}\left(1-\delta_{l, 1}\right)\right. \\
&+\left.\{u(l+1, m)-u(l, m)\}\left(1-\delta_{l, N_{1}}\right)\right] \\
&+ K_{2}\left[\{u(l, m-1)-u(l, m)\}\left(1-\delta_{m, 1}\right)\right. \\
&\left.+\{u(l, m+1)-u(l, m)\}\left(1-\delta_{m, N_{2}}\right)\right], \\
&-\mathbf{1 3 9}
\end{aligned}
$$


where $K_{1}$ and $K_{2}$ are force-constants for different directions. The equation (4.1) is equivalent to the homogeneous equation

$$
\begin{aligned}
-M \omega^{2} u(l, m)= & K_{1}[u(l-1, m)-2 u(l, m)+u(l+1, m)] \\
& +K_{2}[u(l, m-1)-2 u(l, m)+u(l, m+1)]
\end{aligned}
$$

with boundary conditions

$$
\begin{aligned}
& u(0, m)=u(1, m), \\
& u\left(N_{1}+1, m\right)=u\left(N_{1}, m\right), \\
& u(l, 0)=u(l, 1),
\end{aligned}
$$

and

$$
u\left(l, N_{2}+1\right)=u\left(l, N_{2}\right), \quad 1 \leqq l \leqq N_{1}, 1 \leqq m \leqq N_{2} .
$$

Define, as in $\S 1$, the state vector $x(l, m)$ and the transfer matrix $T$ by

$$
x(l, m) \equiv\left(\begin{array}{l}
u(l, m) \\
u(l-1, m)
\end{array}\right),
$$

and

$$
T(l, m) \equiv\left(\begin{array}{cr}
\Gamma(l, m) & -1 \\
1 & 0
\end{array}\right)
$$

where

$$
\begin{aligned}
& \Gamma(l, m) \equiv 2-\left[M(l, m) \omega+K_{2} \Delta^{2}(l)\right] / K_{1}, \\
& \Delta^{2}(l) u(l, m) \equiv u(l, m-1)-2 u(l, m)+u(l, m+1) .
\end{aligned}
$$

Then we can write Eqs. (4.2) in vector-matrix forms:

$$
\begin{aligned}
& x(l+1, m)=T(l, m) x(l, m), \\
& x(1, m)=\left(\begin{array}{l}
1 \\
1
\end{array}\right) u(1, m), \\
& x\left(N_{1}+1, m\right)=\left(\begin{array}{l}
1 \\
1
\end{array}\right) u\left(N_{1}, m\right), \\
& x(l, 0)=x(l, 1), \\
& x\left(l, N_{2}+1\right)=x\left(l, N_{2}\right), \\
& 1 \leqq l \leqq N_{1}, \quad 1 \leqq m \leqq N_{2} .
\end{aligned}
$$

By the use of Eq. (4.5 a), we obtain the equation

$$
x(l, m)=S x(1, m) \text {, }
$$

where 


$$
S \equiv T(l-1, m) T(l-2, m) \cdots T(1, m) .
$$

In the matrix elements of $S$, there appear products of the operators $d^{2}(l)$. In reference (1) it was noted that, in calculating the matrix elements of $S$, we may disregard the dependence of $J^{2}(l)$ on $l$ and treat the products of $J^{2}(l)$ 's (which should be regarded in general as operating on $x(l, m)$, not merely on $u(l, m))$ as higher-order difference operators in the usual sense, if

$$
\begin{aligned}
T(l-2, m-1) & =T(l-2, m)=T(l-2, m+1), \\
T(l-3, m-2) & =T(l-3, m-1)=T(l-3, m) \\
& =T(l-3, m+1)=T(l-3, m+2), \\
T(l-4, m-3) & =T(l-4, m-2) \cdots=T(l-4, m+3), \\
& \ldots \ldots \\
& \cdots \cdots
\end{aligned}
$$

The proof of this fact will be given in the Appendix. It means that the equation of motion must be the same along each line of constant $l$ in the triangular region indicated in Figure 1. This region may be called the "domain of dependence" of $x(l, m)$ in the sense that the value of $x(l, m)$ should be influenced by any change in initial value $x(1, m)$ or in the structure of the lattice (values of masses and force constants) in this region.

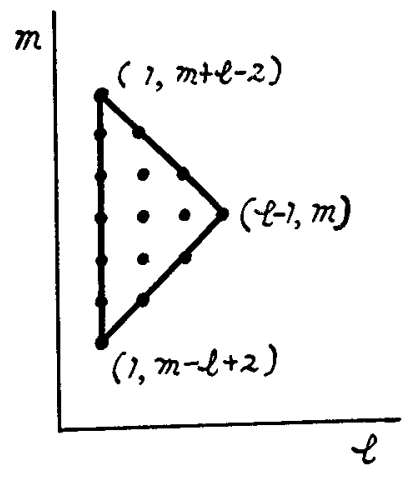

Fig. 1.

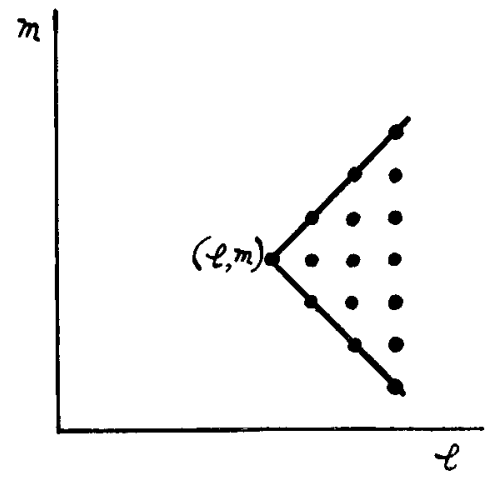

Fig. 2.

The dependence of $J^{2}(l)$ on $l$ cannot be disregarded in $S$ if there are impurities or edges in this domain of dependence, except for the case in which these affect each line of constant $l$ uniformly. If there is an isotopic impurity at $(l, m)$, on the other hand, the equations of motion become different from these for the regular lattice in the region indicated in Figure 2. This region may be called the "region of influence" of the isotope at $(l, m)$. In such a 
region of influence, the dependence of $J^{2}(l)$ on $l$ cannot be neglected in the transfer matrices.

In the present case the lines $m=1$ and $m=N_{2}$ are the edges of the lattice. In view of the above considerations, the values of $x(l, m)$ in the region $A$

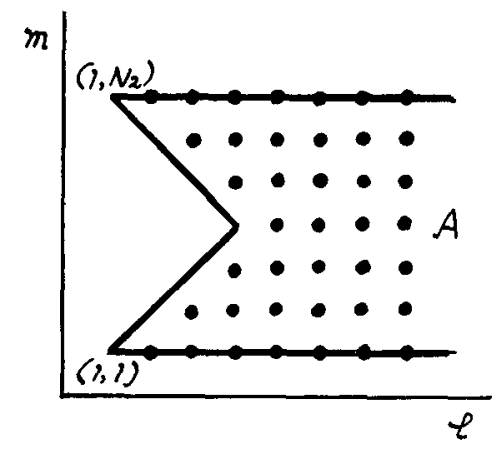

Fig. 3. indicated in Fig. 3 cannot be calculated from $x(1, m), m=1,2, \cdots, N_{2}$, by regarding the $J^{2}(l)$ 's in $S$ as independent of $l$. This situation makes the calculation much more involved compared to the case of cyclic boundary condition. In order to avoid such a complications, we use the following artifice. Let us imagine the lattice extended indefinitely towards both $m$-directions, but impose the condition that displacements should be symmetric with regard to the lines $m=1 / 2$ and $m=N_{2}$ $+1 / 2$. In other words, we imagine that there exist an infinite number of mirror images with respect to the two lines of the original finite lattice. This amounts to requiring the same symmetry to both the values of the initial vectors $x(1, m)$ and the equations of motion for all lattice points. Moreover, the above symmetry requirement with regard to two lines may be replaced by the simultaneous requirements of the symmetry with regard to one of the lines and of a cyclic condition with the period $2 N_{2}$. In the system composed of all the mirror images and the original system, the above condition of uniformity of the equation of motion along the $m$-direction is satisfied so that it is no more necessary to bother about the complications due to the $l$-dependence of $\Delta^{2}(l)$ 's. Moreover, the boundary conditions of free edges (Eq. $(4.5 \mathrm{c}))$ are automatically satisfied in this system. The extra lattices are not connected dynamically to the original lattice, its edges being left free.

The above artifice of introducing the mirror images of the original system is evidently analogous to the "method of image" in the theory of the partial differential equation. In what follows we use this method throughout, so that we may drop the argument $l$ of $d^{2}(l)$ everywhere.

In order to satisfy the above-mentioned requirements the initial displacement $u(1, m)$ must be expanded as

$$
u(1, m)=\sum_{\mu=0}^{2 N_{2}-1} c_{\mu} Z_{\mu}(m),
$$

where

$$
Z_{\mu l}(m)=\exp \left\{i(2 m-1) \chi_{\mu \prime}\right\}
$$


and

$$
\chi_{i l}=\mu \pi / 2 N_{2}, \quad \mu=0,1, \cdots, 2 N_{2}-1,
$$

since it should be periodic with the period $2 N_{2}$.

The symmetry with respect to the line $m=N_{2}+1 / 2$,

$$
u(1, m)=u\left(1,2 N_{2}-m+1\right),
$$

requires that

$$
c_{\mu}=-c_{2 N_{2}-\mu} .
$$

Especially

$$
c_{N_{2}}=0 \text {. }
$$

Further, the value of displacements must be real. From this it follows that

$$
c_{0}=c_{0}{ }^{*} \text { and } \quad c_{\mu}=-c_{2 N_{2}-\mu} * \text {. }
$$

Using Eq. (4.6d), these relations become

$$
c_{\mu}=c_{\mu}^{*}
$$

Then it is clear that only $N_{2}$ real coefficients $c_{\mu}$ 's, $\mu=0, \cdots, N_{2}-1$, are independent of each another.

With the initial value $u(1, m)$ given by Eq. (4.6a), the equation to be solved is

$$
\begin{aligned}
& x(l+1, m)=T x(l, m), \\
& \left\{\begin{array}{l}
x(1, m)=\left(\begin{array}{l}
1 \\
1
\end{array}\right) u(1, m), \\
x\left(N_{1}+1, m\right)=\left(\begin{array}{l}
1 \\
1
\end{array}\right) u\left(N_{1}, m\right), \quad 1 \leqq l \leqq N_{1} \text { and }-\infty<m<\infty .
\end{array}\right.
\end{aligned}
$$

The transfer matrix can be transformed to the diagonal form:

$$
T=P \Lambda P^{-1},
$$

where

$$
\begin{aligned}
& A=\left(\begin{array}{ll}
A_{1} & 0 \\
0 & A_{1}
\end{array}\right), \\
& P=\left(\begin{array}{ll}
A_{1} & \Lambda_{2} \\
1 & 1
\end{array}\right) \\
& P^{-1}=\frac{1}{\Lambda_{1}-\Lambda_{2}}\left(\begin{array}{rr}
1 & -\Lambda_{2} \\
-1 & \Lambda_{2}
\end{array}\right)
\end{aligned}
$$


and

$$
A_{2}=\left\{\Gamma \pm\left(\Gamma^{2}-4\right)^{\frac{1}{2}}\right\} / 2
$$

The $l$-th power of $T$ is given by

$$
T^{l}=\frac{1}{\Lambda_{1}-\Lambda_{2}}\left(\begin{array}{ll}
\Lambda_{1}^{l+1}-\Lambda_{2}^{l+1} & -\left(A_{1}^{l}-A_{2}^{l}\right) \\
\Lambda_{1}^{l}-\Lambda_{2}^{l} & -\left(\Lambda_{1}^{l-1}-A_{2}^{l-1}\right)
\end{array}\right) .
$$

From Eqs. (4.4b) and (4.8e), it is seen that the matrix element $\Gamma$ and consequently $A_{2}$ are operators involving the operator $\Delta^{2}$.

The functions $Z_{n}(m),\left(\mu=0,1, \cdots, 2 N_{2}-1\right)$, in the expansion (4.6a) are the eigenfunctions of the operator $J^{2}$, as well as of $\Gamma$ and $A_{2}$. Consequently, if we put

$$
M \omega^{2}=4 K_{1} \sin ^{2} \theta_{\mu}+4 K_{2} \sin ^{2} \chi_{\mu}
$$

and

then

$$
\theta_{2 N_{2} \cdots \mu}=\theta_{\mu}, \quad \mu=0,1, \cdots, N_{2},
$$

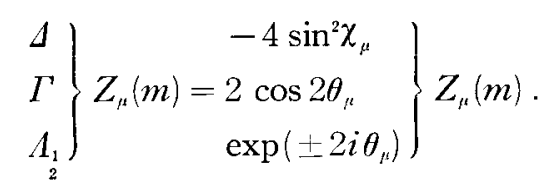

From Eq. (4.7 a) we obtain

$$
x\left(N_{1}+1, m\right)=T^{N_{1}} x(1, m) \quad \text { for all } m .
$$

Combined with Eqs. $(4.7 \mathrm{~b})$ and (4.9), this reduces to

$$
\begin{aligned}
& \left(\begin{array}{l}
1 \\
1
\end{array}\right) u\left(N_{1}, m\right)=T^{N_{1}}\left(\begin{array}{l}
1 \\
1
\end{array}\right) u(1, m) \\
& =\frac{1}{A_{1}-A_{2}}\left(\begin{array}{c}
A_{1}^{N_{1}+1}-A_{2}^{N_{1}+1}-\left(\Lambda_{1}^{N_{1}}-A_{2}^{N_{1}}\right) \\
\Lambda_{1}^{N_{1}}-A_{2}^{N_{1}}-\left(\Lambda_{1}^{N_{1}-1}-\Lambda_{2}^{N_{1}-1}\right)
\end{array}\right) u(1, m) .
\end{aligned}
$$

If we substitute Eqs. (4.6a) and (4.11) into Eq. (4.13), it follows that

$$
\left(\begin{array}{l}
1 \\
1
\end{array}\right) u\left(N_{1}, m\right)=\sum_{\mu=n}^{2 N_{2}-1} c_{\mu}\left(\begin{array}{l}
S_{\mu}\left(N_{1}+1\right)-S_{\mu}\left(N_{1}\right) \\
S_{\mu}\left(N_{1}\right)-S_{\mu}\left(N_{1}-1\right)
\end{array}\right) Z_{\mu}(m)
$$

where

$$
S_{\mu}(l)=\sin 2 l \theta_{n} / \sin 2 \theta_{\mu}=S_{2 N_{2}-\mu}(l)
$$

From Eq. (4.14a), we get

$$
\sum_{n=0}^{2 N_{2}-1} c_{\mu} A_{\mu} Z_{\mu}(m)=0 \quad \text { for all } m,
$$

where 


$$
A_{\mu}=S_{\mu}\left(N_{1}+1\right)-2 S_{\mu}\left(N_{1}\right)+S_{\mu}\left(N_{1}-1\right)
$$

By the use of the orthogonality relation of the eigenfunctions $Z_{\mu}(m)$,

$$
\sum_{m=0}^{2 N_{2}-1} Z_{\mu}{ }^{*}(m) Z_{\nu}(m)=2 N_{2} \delta_{\mu \nu},
$$

Eq. (4.15a) is reduced to

$$
c_{\mu} A_{\mu}=0, \quad \mu=0,1, \cdots, N_{2}-1 \text {. }
$$

The condition for Eq. (4.17) to have a non-trivial solution is

$$
\prod_{\mu=0}^{N_{2}-1} A_{\mu}=0 \text {. }
$$

Using Eqs. (4.15b) and (4.14b), we get

$$
\prod_{n=0}^{N_{2}-1} \sin 2 N_{1} \theta_{\mu} \tan \theta_{\mu}=0 \text {. }
$$

Consequently, we have

$$
\theta_{\mu}=\kappa \pi / 2 N_{1}, \quad \mu=0,1, \cdots, N_{2}-1 \text { and } \kappa=0,1, \cdots, N_{1}-1 .
$$

Introducing this into Eq. (4.10), we get the eigenfrequencies of the square lattice with free edges:

$$
M \omega_{0}^{2}(\kappa, \mu)=4 K_{1} \sin ^{2} \varphi_{\kappa}+4 K_{2} \sin ^{2} \chi_{\mu},
$$

where

$$
\varphi_{\kappa}=\kappa \pi / 2 N_{1}, \quad \kappa=0,1, \cdots, N_{1}-1
$$

and

$$
\chi_{\mu}=\mu \pi / 2 N_{2}, \quad \mu=0,1, \cdots, N_{2}-1 .
$$

Consulting Eqs. (4.12) (4.14), we can obtain the normal mode belonging to the eigenfrequency $\omega_{0}\left(\kappa^{\prime}, \mu^{\prime}\right)$ :

$$
\begin{aligned}
x(l, m) & =T^{l-1} x(1, m) \\
& =\sum_{\mu=0}^{2 N_{z}-1} c_{\mu}\left(\begin{array}{l}
S_{\mu}(l)-S_{\mu}(l-1) \\
S_{\mu}(l-1)-S_{\mu}(l-2)
\end{array}\right) Z_{\mu}(m),
\end{aligned}
$$

where the parameters $\theta_{\mu}$ 's are defined by Eq. (4.10), i.e.,

$$
\begin{aligned}
M \omega_{0}^{2}\left(\kappa^{\prime}, \mu^{\prime}\right) & =4 K_{1} \sin ^{2} \varphi_{\kappa^{\prime}}+4 K_{2} \sin ^{2} \chi_{\mu^{\prime}} \\
& =4 K_{1} \sin ^{2} \theta_{\mu}+4 K_{2} \sin ^{2} \chi_{\mu}
\end{aligned}
$$

and

$$
\begin{array}{r}
\theta_{2 N_{8}-\mu}=\theta_{\mu}, \quad \mu=0,1, \cdots, N_{2}-1 . \\
-\mathbf{1 4 5}-
\end{array}
$$


If we assume that the accidental degeneracy does not occur in the frequency spectrum, that is, the frequencies specified by different pairs of $\kappa$ and $\mu$ may not be identical, then all the coeficients $c_{\mu}$ 's except only one $c_{\mu^{\prime}}$, must vanish on account of Eq. (4.17). Consequently, we get

$$
\begin{aligned}
& u(l, m)= \begin{cases}c_{\mu^{\prime}}\left[S_{\mu^{\prime}}(l)-S_{\mu^{\prime}}(l-1)\right]\left[Z_{\mu^{\prime}}(m)-Z_{2 N_{2}-\eta^{\prime}}(m)\right], & \mu^{\prime} \neq 0 \\
c_{0}\left[S_{\mu^{\prime}}(l)-S_{\mu^{\prime}}(l-1)\right], & \mu^{\prime}=0\end{cases} \\
& =\left(2-\delta_{\mu^{\prime} 0}\right) c_{\mu^{\prime}} \sec \varphi_{\kappa^{\prime}} \cos \left\{(2 l-1) \varphi_{k^{\prime}}\right\} \cos \left\{(2 m-1) \chi_{\mu^{\prime}}\right\} \text {. }
\end{aligned}
$$

From this result it is clear that there does not come out any surface mode.

In view of the fact that the partial difference equation (4.2a) is of the type which allows the "separation of variables", the results obtained above are what may be naturally expected.

\section{Two-Dimensional Monatomic Lattice with Isotopic Impurities on one of the Edges}

At first consider a finite monatomic square lattice with an isotopic impurity atom at a lattice point $\left(N_{1}, j\right)$ on its edge.

When we write the equation of motion in the vector-matrix form as in $\S 4$, only one transfer matrix $T^{\prime}$ connecting $x\left(N_{1}, j\right)$ with $x\left(N_{1}+1, j\right)$ comes out to be different from that of the regular lattice $T$. If the extra lattice is introduced in order to avoid the complication pertaining to the products of difference operators, the impurity transfer-matrix $T^{\prime}$ appears at the points which meet the symmetry requirements described in $\S 4$ :

$$
x\left(N_{1}+1, m\right)=T^{\prime} x\left(N_{1}, m\right), \quad m=j, 2 N_{2}-j+1, \cdots
$$

where

$$
\begin{aligned}
& T^{\prime}=\left(\begin{array}{rr}
\Gamma^{\prime} & -1 \\
1 & 0
\end{array}\right) \\
& \Gamma^{\prime \prime}=2-\left(M^{\prime} \omega^{2}+K_{2} \Delta^{2}\right) / K_{1}
\end{aligned}
$$

and $M^{\prime}$ is the impurity mass.

If we introduce the parameters $Q$ and $\delta$ by

$$
Q=\left(M^{\prime}-M\right) / M, \quad \delta=Q M \omega^{2} / K_{1},
$$

then $T^{\prime}$ becomes

$$
T^{\prime}=T-\left(\begin{array}{ll}
\delta & 0 \\
0 & 0
\end{array}\right) .
$$


For $m \neq j, 2 N_{2}-j+1, \cdots$, we get, as before,

$$
x\left(N_{1}+1, m\right)=T^{N_{1}} x(1, m), \quad m \neq j, 2 N_{2}-j+1, \cdots,
$$

which turns out to be

$$
\sum_{\mu-1}^{2 N_{2}-1} c_{\mu} A_{\mu} Z_{\mu}(m)=0, \quad m \neq j, 2 N_{2}-j+1, \cdots
$$

For $m=j$, we obtain from Eqs. (5.1a) and (5.2b)

$$
x\left(N_{1}+1, m\right)=T^{\prime} T^{N_{1}-1} x(1, m), \quad m=j, 2 N_{2}-j+1, \cdots .
$$

This is seen to be equivalent to

$$
\sum_{\mu=0}^{2 N_{2}-1} c_{\mu}\left(A_{\mu}-\delta B_{\mu}\right) Z_{\mu}(m)=0
$$

where

$$
A_{\mu}=S_{\mu}\left(N_{1}+1\right)-2 S_{\mu}\left(N_{1}\right)+S_{\mu}\left(N_{1}-1\right)
$$

and

$$
B_{\mu}=S_{\mu}\left(N_{1}\right)-S_{\mu}\left(N_{1}-1\right) .
$$

Using the orthogonality relation (4.16) and the symmetry condition $(4.6 \mathrm{~d})$, we obtain from Eqs. (5.4) and (5.6a) a system of equations:

$$
\begin{gathered}
c_{\mu} A_{\mu}-\cos \left\{(2 j-1) \chi_{\mu}\right\} \frac{\delta}{N_{2}} \sum_{\nu=0}^{N_{2}-1}\left(2-\delta_{\nu 0}\right) c_{\nu} B_{\nu} \cos \left\{(2 j-1) \chi_{\nu}\right\}=0, \\
\mu=0,1, \cdots, N_{2}-1 .
\end{gathered}
$$

The condition for the existence of a non-trivial solution is

$$
D \equiv \prod_{\mu=0}^{N_{2}-1} A_{\mu}-\frac{\delta}{N_{2}} \sum_{\mu=0}^{N_{2}-1}\left(2-\delta_{\mu 0}\right) \cos ^{2}\left\{(2 j-1) \chi_{\mu}\right\} B_{\mu} \prod_{\nu \neq \mu} A_{\nu}=0 .
$$

Let us at first seek the eigenfrequencies which are not affected by the presence of impurity. If there is no accidental degeneracy in the unperturbed spectrum, then the unperturbed eigenfrequency satisfying the equation $A_{u}=0$ remains to be eigenfrequency if and only if $\cos \left\{(2 j-1) \chi_{\mu}\right\}=0$, since the equations $A_{\mu}=0$ and $B_{\mu}=0$ cannot be satisfied simultaneously. Thus the unperturbed normal mode which has a node at the impurity site remains to be a normal mode. In order to get other eigenfrequencies, it is convenient to rewrite Eq. (5.5) as follows. Since none of the quantities $A_{\mu}$ vanishes for these frequencies, it follows that

$$
1-\frac{\delta}{N_{2}} \sum_{\mu=0}^{N_{2}-1}\left(2-\delta_{\mu 0}\right) \cos ^{2}\left\{(2 j-1) \chi_{\mu}\right\} \frac{B_{\mu}}{A_{\mu}}=0
$$

or 


$$
1-\frac{\delta}{N_{2}} \sum_{n=0}^{2 N_{2}-1} \cos ^{2}\left\{(2 j-1) \chi_{\mu}\right\} \frac{B_{\mu}}{A_{\mu}}=0 .
$$

From Eqs. $(5.6 \mathrm{~b}, \mathrm{c})$, we obtain

$$
\begin{aligned}
\frac{B_{\mu}}{A_{\mu}} & =-\frac{\cos \left(2 N_{1}-1\right) \theta_{\mu^{\prime}}}{2 \sin \theta_{\mu} \sin 2 N_{\mathrm{s}} \theta_{\mu}} \\
& =-\left(1+\cot \theta_{\mu} \cot 2 N_{\mathrm{l}} \theta_{\mu}\right) / 2 .
\end{aligned}
$$

Substituting Eq. (5.10b) into Eq. $(5.9 \mathrm{~b})$, we get

$$
1+\frac{\delta}{2}\left\{1+\frac{1}{N_{2}} \sum_{\mu=0}^{2 N_{2}-1} \cos ^{2}\left\{(2 j-1) \chi_{\mu}\right\} \cot \theta_{\mu} \cot 2 N_{1} \theta_{\mu}\right\}=0,
$$

where we used a simple relation

$$
\sum_{\mu=0}^{2 N_{2}-1} \cos ^{2}\left\{(2 j-1) \chi_{\mu}\right\}=N_{2} \text {. }
$$

From these equations we can readily obtain the following conclusions: None of these eigenfrequencies can be equal to unperturbed ones. There is, however, a one-to-one correspondence between them, and the corresponding pair coincide with each other when the impurity disappears. All the perturbed eigenfrequencies are larger or smaller than the corresponding unperturbed frequencies, according as $Q<0$ (light impurity) or $Q>0$ (heavy impurity). The former cannot however exceed the neighboring unperturbed frequencies.

From these results it is clear that only one eigenfrequency may become extra-band frequency which is larger than any unperturbed eigenfrequencies in the case of $Q<0$. In what follows let us discuss this particular frequency. Defining the variables $\varepsilon_{\mu}$ 's by

$$
\theta_{\mu}=\pi / 2+i \varepsilon_{\mu}, \quad \mu=0,1, \cdots, N_{2}-1,
$$

we get

$$
\cot \theta_{\mu} \cot 2 N_{1} \theta_{\mu} \cong-\tanh \varepsilon_{\mu},
$$

where we assumed that

$$
\operatorname{coth} 2 N_{1} \varepsilon_{\mu} \cong 1, \quad \text { i.e., } \quad \varepsilon_{\mu} \gg 1 / N_{1} \text {. }
$$

This assumption is necessary in order that the normal mode corresponding to the extra-band frequency is spatially localized around the impurity. The equation (5.11) is then transformed to

$$
\begin{gathered}
1+\frac{Q M \omega^{2}}{2 K_{1}}\left\{1-\frac{1}{N_{2}} \sum_{\mu=0}^{2 N_{2}-1} \cos ^{2}\left\{(2 j-1) \chi_{\mu}\right\} \tanh \varepsilon_{\mu}\right\}=0 . \\
-\mathbf{1 4 8}-
\end{gathered}
$$


From Eq. (4.10) and (5.12) it follows that

$$
\tanh \varepsilon_{p}=\left(\frac{M \omega^{2}-4 K_{1}-4 K_{2} \sin ^{2} \chi_{\mu}}{M \omega^{2}-4 K_{2} \sin ^{2} \chi_{\mu}}\right)^{\frac{1}{2}}
$$

To determine the critical value of $Q$ below which the extra-band frequency appears, put $M \omega^{2}=4 K_{1}+4 K_{2}$ in Eqs. (5.13). After simple calculations, it follows that

$$
1+\left(\frac{2 Q_{c}}{1-p^{2}}\right)\left\{1-\frac{1}{N_{2}} \sum_{\mu=0}^{2 N_{2}-1} \frac{p\left|\cos \chi_{\mu}\right|}{\sqrt{1-p^{2} \sin ^{2} \chi_{\mu}}} \cos ^{2}\left\{(2 j-1) \chi_{\mu}\right\}\right\}=0,
$$

where

$$
0 \leqq p=\sqrt{K_{2} /\left(K_{1}+K_{2}\right)}<1 .
$$

The critical value $Q_{0}$ depends on the position of the impurity. Only two special cases $\mathrm{A}$ and $\mathrm{B}$ are examined in the following.

Case A. First we examine the case in which the impurity is located at the center of the edge $\left(2 j-1=N_{2}\right)$. Using the relation

$$
\begin{aligned}
\cos ^{2}\left\{(2 j-1) \chi_{\mu}\right\} & =\cos ^{2}(\mu \pi / 2) \\
& = \begin{cases}1, & \mu=0,2, \cdots, 2 N_{2}-2 \\
0, & \mu=1,3, \cdots, 2 N_{2}-1,\end{cases}
\end{aligned}
$$

we can reduce Eq. $(5.14 \mathrm{a})$ to

$$
1+\left(\frac{2 Q_{c}}{1-p^{2}}\right)\left\{1-\frac{1}{N_{2}} \sum_{\mu}^{\prime} \frac{p\left|\cos \chi_{\mu}\right|}{\left(1-p^{2} \sin ^{2} \chi_{\mu}\right)^{\frac{1}{2}}}\right\}=0
$$

where the prime indicates the summation over even $\mu$ only. If $N_{2}$ is large, so that the sum may be replaced by an integral, Eq. (5.16) becomes

$$
1+\left(\frac{2 Q}{1-p^{2}}\right)\left\{1-\frac{2}{\pi} \int_{0}^{\frac{\pi}{2}} \frac{p \cos \chi \mathrm{d} \chi}{\left(1-p^{2} \sin ^{2} \chi\right)^{\frac{1}{2}}}\right\}=0 \text {. }
$$

Using the well-known relation

$$
\int_{0}^{\frac{\pi}{2}} \frac{p \cos \chi d \chi}{\left(1-p^{2} \sin ^{2} \chi\right)^{\frac{1}{2}}}=\sin ^{-1} p
$$

we get

$$
Q_{c}=-\frac{1}{2} \frac{1-p^{2}}{1-(2 / \pi) \sin ^{-1} p} .
$$

The variation of $Q_{c}$ as the function of the parameter $p$ is shown in Fig. 4 (curve A). 
Case B. In a case where the impurity is located at the corner point $(j=1)$, we get the equation

$$
1+\left(\frac{2 Q_{c}}{1-p^{2}}\right)\left\{1-\frac{1}{N_{2}} \sum_{\mu=0}^{2 N_{2}-1} \frac{p \cos ^{3} \chi_{\mu}}{\left(1-p^{2} \sin ^{2} \chi_{\mu}\right)^{\frac{1}{2}}}\right\}=0 .
$$

For the large number $N_{2}$ it becomes, as before,

$$
1+\left(\frac{2 Q_{c}}{1-p^{2}}\right)\left\{1-\frac{4}{\pi} \int_{0}^{\frac{\pi}{2}} \frac{p \cos ^{3} \chi d \chi}{\left(1-p^{2} \sin ^{2} \chi\right)^{\frac{1}{2}}}\right\}=0 .
$$

Using the well-known relation

$$
\int_{0}^{\frac{\pi}{2}} \frac{\cos ^{3} \chi d \chi}{\left(1-p^{2} \sin ^{2} \chi\right)^{\frac{1}{2}}}=\frac{1}{2 p^{2}}\left\{\left(1-p^{2}\right)-\frac{1}{p}\left(1-2 p^{2}\right) \sin ^{-1} p\right\},
$$

we obtain

$$
Q_{c}=-\frac{1}{2} \frac{1-p^{2}}{1-(2 / \pi)\left\{\left(1-p^{2}\right) \frac{1}{2} / p-\left(1-2 p^{2}\right) / p^{2} \cdot \sin ^{-1} p\right\}} .
$$

Fig. 4 (curve B) shows the variation of $Q_{c}$ for this case as the function of $p$.

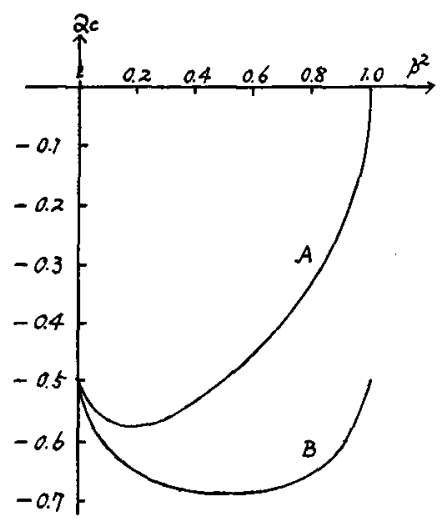

Fig. 4.

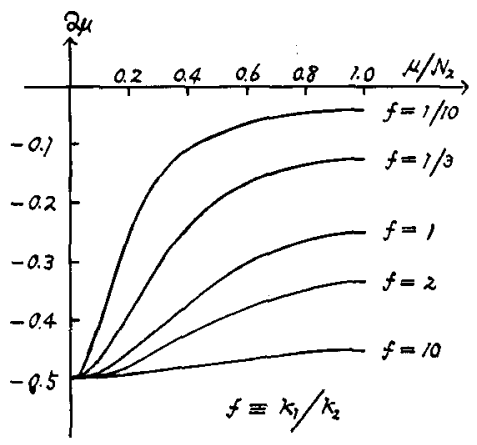

Fig. 5 .

When $K_{2}<K_{1}$, the value of $p^{2}$ is smaller than $\frac{1}{2}$, and becomes zero as $K_{2} \rightarrow 0$. This limit corresponds to the case of independent linear chains extended in $l$-direction. Hence it is natural that here both curves in Fig. 4 give the same value $Q_{c}=-\frac{1}{2}$, which moreover coincides with that obtained in $\S 2$ for the linear lattice. On the other hand, $p^{2}$ is larger than $\frac{1}{2}$ when $K_{2}>K_{1}$, and becomes unity as $K_{1} \rightarrow 0$. This limit corresponds to the case of independent linear chains extended in $m$-direction. Since in Case $\mathrm{B}$ the position of the impurity is symmetric with respect to two directions, it is a matter of course 
that the curve $\mathrm{B}$ is symmetric about its center, and gives $Q_{c}=-\frac{1}{2}$ for $p^{2}=1$. On the contrary the position of the impurity is asymmetric with respect to two directions, in Case $\mathrm{A}$, and the curve $\mathrm{A}$ gives a different value $Q_{0}=0$. This means that for the linear chain with an impurity at its center, the localized mode appears as soon as its mass becomes lighter than that of host atoms. That the curve A gives higher values of $Q_{c}$ in the right half than in the left half of the figure means that, if the force constant in the longitudinal direction is larger than that in the lateral direction, as is usually the case, the vibration in the direction of the edge is more easily localized than that in the direction normal to the edge, for the impurity situated at the center of the edge.

For the mode corresponding to the extra-band frequency of the twodimensional lattice with an impurity on the edge, it is almost clear, from the arguments of the previous paper ${ }^{1)}$, that it is composed of the partial waves which are localized in $l$-direction. In Case B the same argument applies also to $m$-direction, on account of the symmetry mentioned above. The mode is thus localized in both directions. Although in Case A such an argument based on the symmetry is not valid, it is highly probable that the mode is also localized around the impurity site.

Next let us consider a two-dimensional lattice, one of the edges of which $\left(l=N_{1}\right)$ is entirely composed of impurities of the same kind. We have then, in the same way as above,

$$
x\left(N_{1}+1, m\right)=T^{\prime} T^{N_{1}-1} x(1, m),
$$

which reduces to

$$
\sum_{\mu=0}^{2 N_{2}-1} c_{\mu}\left(A_{\mu}-\delta B_{\mu}\right) Z_{\mu}(m)=0, \quad 1 \leqq m \leqq 2 N_{2} .
$$

The condition for existence of a non-trivial solution is

$$
D \equiv \prod_{\mu=0}^{N_{2}-1}\left(A_{\mu}-\delta B_{\mu}\right)=0 \text {. }
$$

Consequently,

$$
A_{n}-\delta B_{\mu}=0
$$

or

$$
\begin{array}{r}
\tan \theta_{\mu} \sin 2 N_{1} \theta_{\mu}\left\{1+(\delta / 2)\left(1+\cot \theta_{\mu} \cot 2 N_{1} \theta_{\mu}\right)\right\}=0, \\
\mu=0,1, \cdots, N_{2}-1 .
\end{array}
$$

The roots of the first factor of this equation are quite similar to those in the one-dimensional case. For the roots of the second factor, however, the situation 
is remarkably different. There may be complex roots for some values of $Q$. The eigenfrequencies corresponding to these roots may lie both inside and outside the frequency band of the unperturbed spectrum. In fact, substituting $\theta_{\mu}=\pi / 2+i \varepsilon_{\mu}\left(\varepsilon_{\mu}>0\right)$ into the second factor of Eq. (5.28), we obtain

$$
Q=-\frac{1}{2} \frac{\exp \left(\varepsilon_{\mu}\right) \cosh \varepsilon_{\mu}}{\cosh ^{2} \varepsilon_{\mu}+\left(K_{2} / K_{1}\right) \sin ^{2} \chi_{\mu}} .
$$

From this we can find the critical values $Q_{k}$ for the appearance of the complex roots $\theta_{\mu}$ :

$$
Q_{\mu}=-\frac{1}{2} \frac{K_{1}}{K_{1}+K_{2} \sin ^{2} \chi_{\mu}} .
$$

It follows that

$$
-\frac{1}{2}=Q_{0}<Q_{1}<\cdots<Q_{N_{2}-1}<-\frac{1}{2}\left(1-p^{2}\right) .
$$

This means that the eigenfrequencies corresponding to the complex roots may appear more easily from the high-frequency region than from the low-frequency region of the band. The variation of some of $Q_{\mu}$ 's as functions of the parameter $f \equiv K_{1} / K_{2}=\left(1-p^{2}\right) / p^{2}$ is shown in Fig. 5 .

It may readily be shown, in the same way as in $\S 2$, that the normal modes corresponding to these eigenfrequencies damp exponentially as the distance from the edge composed of impurities increases:

$$
u(l, m) \cong(-1)^{N_{1}-l} \exp \left\{-2\left(N_{1}-l\right) \varepsilon_{\mu}\right\} u\left(N_{1}, m\right) .
$$

\section{Three-Dimensional Regular Monatomic Lattices with Free Boundaries}

In the same way as in $\S 4$, it is readily shown that the generalized transfermatrix method is applicable to a three-dimensional regular monatomic lattice with free boundaries.

The equation of motion for one of the components of atomic displacement $u(l, m, n)$ at a lattice point $(l, m, n)$ can be written in the form:

$$
\begin{gathered}
-M \omega^{3} u(l, m, n)=K_{1}\left[\{u(l-1, m, n)-u(l, m, n)\}\left(1-\delta_{l, l}\right)\right. \\
\left.+\{u(l+1, m, n)-u(l, m, n)\}\left(1-\delta_{l, N_{1}}\right)\right] \\
+K_{2}\left[\{u(l, m-1, n)-u(l, m, n)\}\left(1-\delta_{m, 1}\right)\right. \\
\left.+\{u(l, m+1, n)-u(l, m, n)\}\left(1-\delta_{m, N_{2}}\right)\right] \\
-\mathbf{1 5 2}-
\end{gathered}
$$


Some Applications of the Method of Transfer-Matrix to the Vibration of Lattices

$$
\begin{aligned}
+K_{3} & {\left[\left\{u(l, m, n-1-u(l, m, n)\}\left(1-\delta_{n, 1}\right)\right.\right.} \\
+ & \left.\{u(l, m, n+1)-u(l, m, n)\}\left(1-\delta_{n, N_{3}}\right)\right] .
\end{aligned}
$$

The equation $(6.1)$ is equivalent to the homogeneous equation

$$
\begin{aligned}
-M \omega^{2} u(l, m, n) & =K_{1}[u(l-1, m, n)-2 u(l, m, n)+u(l+1, m, n)] \\
& +K_{2}[u(l, m-1, n)-2 u(l, m, n)+u(l, m+1, n)] \\
& +K_{3}[u(l, m, n-1)-2 u(l, m, n)+u(l, m, n+1)],
\end{aligned}
$$

with boundary conditions

$$
\begin{aligned}
& \left\{\begin{array}{l}
u(0, m, n)=u(1, m, n), \\
u\left(N_{1}+1, m, n\right)=u\left(N_{1}, m, n\right),
\end{array}\right. \\
& \left\{\begin{array}{l}
u(l, 0, n)=u(l, 1, n), \\
u\left(l, N_{2}+1, n\right)=u\left(l, N_{2}, n\right),
\end{array}\right. \\
& \left\{\begin{array}{l}
u(l, m, 0)=u(l, m, 1), \\
u\left(l, m, N_{3}+1\right)=u\left(l, m, N_{3}\right), \\
1 \leqq l \leqq N_{1}, 1 \leqq m \leqq N_{2}, 1 \leqq n \leqq N_{3} .
\end{array}\right.
\end{aligned}
$$

Introducing, as before, the state vector $x(l, m, n)$ and the transfer matrix $T$ by

$$
x(l, m, n)=\left(\begin{array}{l}
u(l, m, n) \\
u(l-1, m, n)
\end{array}\right)
$$

and

$$
T(l, m, n)=\left(\begin{array}{cc}
\Gamma(l, m, n) & -1 \\
1 & 0
\end{array}\right)
$$

where

$$
\Gamma(l, m, n)=2-\left\{M(l, m, n) \omega^{2}+K_{2} \Delta_{2}^{2}(l, n)+K_{3} \Delta_{3}^{2}(l, m)\right\} / K_{1}
$$

$\Delta_{2}^{2}(l, n) u(l, m, n)=u(l, m-1, n)-2 u(l, m, n)+u(l, m+1, n)$

$\Delta_{3}^{2}(l, m) u(l, m, n)=u(l, m, n-1)-2 u(l, m, n)+u(l, m, n+1)$, 
we can write Eqs. (6.2) in vector-matrix forms:

$$
\begin{aligned}
& x(l+1, m, n)=T(l, m, n) x(l, m, n), \\
& \left\{\begin{array}{l}
x(1, m, n)=\left(\begin{array}{l}
1 \\
1
\end{array}\right) u(1, m, n), \\
x\left(N_{1}+1, m, n\right)=\left(\begin{array}{l}
1 \\
1
\end{array}\right) u\left(N_{1}, m, n\right),
\end{array}\right. \\
& \left\{\begin{array}{l}
u(l, 0, n)=u(l, 1, n), \\
u\left(l, N_{2}+1, n\right)=u\left(l, N_{2}, n\right),
\end{array}\right. \\
& \left\{\begin{array}{l}
u(l, m, 0)=u(l, m, 1), \\
u\left(l, m, N_{3}+1\right)=u\left(l, m, N_{3}\right) .
\end{array}\right.
\end{aligned}
$$

In order to avoid the complication pertaining to the products of difference operators, the extra lattices which satisfy the same symmetry requirements as in $\$ 4$ are introduced both in $m$ - and $n$-directions. Then we can drop the arguments about the difference operators and expand the initial displacement $u(1, m, n)$ in the form:

$$
u(1, m, n)=\sum_{\mu=0}^{2 N_{2}-1} \sum_{\nu=0}^{2 N_{3}-1} c_{\mu \nu} Z_{j \nu}(m, n),
$$

where

$$
\begin{aligned}
& Z_{\mu \nu}(m, n)=\exp \left[i\left\{(2 m-1) \chi_{\mu}+(2 n-1) \phi_{\nu}\right\}\right], \\
& \left\{\begin{array}{l}
c_{\mu \nu}=c_{\mu \nu}^{*} . \\
c_{\mu \nu}=-c_{2 N_{2}-\mu \nu}=-c_{\mu 2 N_{3}-\nu}=c_{2 N_{2}-\mu 2 N_{3}-\nu},
\end{array}\right. \\
& \begin{cases}\chi_{\mu}=\mu \pi / 2 N_{2}, & \mu=0,1, \cdots, 2 N_{2}-1, \\
\phi_{\nu}=\nu \pi / 2 N_{3}, & \nu=0,1, \cdots, 2 N_{3}-1 .\end{cases}
\end{aligned}
$$

With the initial value $u(1, m, n)$ given by Eq. $(6.5 \mathrm{a})$, the equation to be solved is

$$
\begin{aligned}
& x(l+1, m, n)=T x(l, m, n), \\
& \left\{\begin{aligned}
x(1, m, n)=\left(\begin{array}{l}
1 \\
1
\end{array}\right) u(1, m, n), \\
x\left(N_{1}+1, m, n\right)=\left(\begin{array}{c}
1 \\
1
\end{array}\right) u\left(N_{1}, m, n\right), \\
1 \leqq l \leqq N_{1},-\infty<m, n<\infty .
\end{aligned}\right.
\end{aligned}
$$

The transfer matrix $T$ can be transformed to the same diagonal form as in Eq. (4.8). The relations between the function $Z_{\mu \nu}(m, n)$ and the operators $\Delta_{2}^{2}, \Delta_{3}^{2}, \Gamma$ and $A_{2}^{1}$ which appear in the matrix elements of $T$ are quite similar 
to those in Eq. (4.11). That is, if we put

$$
\begin{aligned}
& M \omega^{2}=4 K_{1} \sin ^{2} \theta_{\mu \nu}+4 K_{2} \sin ^{2} \chi_{\mu}+4 K_{3} \sin ^{2} \phi_{\nu} \\
& \theta_{\mu \nu}=\theta_{2 N_{2}-\mu \nu}=\theta_{\alpha_{2 N_{3}-\nu}}=\theta_{2 N_{2}-\mu}{ }^{2 N_{2}-\nu},
\end{aligned}
$$

it follows that

$$
\left.\left.\begin{array}{c}
\Delta_{2}^{2} \\
J_{3}^{2} \\
\Gamma \\
A_{1}
\end{array}\right\} Z_{\mu \nu}(m, n)=\begin{array}{c}
-4 \sin ^{2} \chi_{\mu} \\
-4 \sin ^{2} \psi_{\nu} \\
2 \cos 2 \theta_{\mu \nu} \\
\exp \left( \pm 2 i \theta_{\mu \nu}\right)
\end{array}\right\} Z_{\mu \nu}(m, n)
$$

To solve the equation of motion we use the method of solution adopted in $\$ 4$ (Eqs. (4.12) (4.15)). We obtain

$$
\sum_{\mu-0}^{2 N_{2}-1} \sum_{\nu=0}^{2 N_{3}-1} c_{\mu \nu} A_{\mu \nu} Z_{\mu \nu}(m, n)=0, \quad \text { for all }(m, n)
$$

where

$$
\begin{aligned}
& A_{\mu \nu}=S_{\mu \nu}\left(N_{1}+1\right)-2 S_{\mu \nu}\left(N_{1}\right)+S_{\mu \nu}\left(N_{1}-1\right) \\
& S_{\mu \nu}(l)=\sin 2 l \theta_{\mu \nu} / \sin 2 \theta_{\mu \nu} .
\end{aligned}
$$

By the use of the orthogonality relation of the functions $Z_{r \nu}(m, n)$,

$$
\sum_{m=1}^{2 N_{2}} \sum_{n=1}^{2 N_{3}} Z_{\mu \nu}^{*}(m, n) Z_{\mu^{\prime} \nu^{\prime}}(m, n)=2 N_{2} 2 N_{3} \delta_{\mu \mu} \delta_{\nu \nu^{\prime}}
$$

the equation $(6.9 \mathrm{a})$ is reduced to

$$
c_{\mu \nu} A_{\mu \nu}=0 \text {. }
$$

The condition for Eq. (6.11) to have a non-trivial solution is

$$
\prod_{(\mu, \nu)=(0,0)}^{\left(N_{\mathrm{a}}-1, N_{3}-1\right)} A_{\mu \nu}=0
$$

or

$$
\prod_{(\mu, \nu)=(0,0)}^{\left(N_{2}-1, N_{3}-1\right)} \sin 2 N_{1} \theta_{\mu \nu} \tan \theta_{\mu \nu}=0 .
$$

Consequently we obtain

$$
\theta_{\mu \nu}=\kappa \pi / 2 N_{1},
$$

where

$$
\kappa=0,1, \cdots, N_{1}-1,
$$




$$
\begin{aligned}
& \mu=0,1, \cdots, N_{2}-1, \\
& \nu=0,1, \cdots, N_{3}-1 .
\end{aligned}
$$

Introducing this into $\mathrm{Eq} .(6.7 \mathrm{a})$, we get the eigenfrequencies of the threedimensional lattice with free boundaries:

$$
M \omega_{0}^{2}(\kappa, \mu, \nu)=4 K_{1} \sin ^{2} \varphi_{x}+4 K_{2} \sin ^{2} \chi_{\mu}+4 K_{3} \sin ^{2} \psi_{\nu},
$$

where

$$
\begin{array}{ll}
\varphi_{\kappa}=\kappa \pi / 2 N_{1}, & \kappa=0,1, \cdots, N_{1}-1, \\
\chi_{\mu}=\mu \pi / 2 N_{2}, & \mu=0,1, \cdots, N_{2}-1, \\
\phi_{\nu}=\nu \pi / 2 N_{3}, & \nu=0,1, \cdots, N_{3}-1 .
\end{array}
$$

If we assume, as in $\$ 4$, that the accidental degeneracy does not occur in the frequency spectrum, we can readily obtain the normal mode belonging to the eigenfrequency $\omega_{0}(\kappa, \mu, \nu)$ :

$$
\begin{aligned}
u(l, m, n) & =\left(2-\delta_{\mu^{0}}\right)\left(2-\delta_{\nu 0}\right) c_{\mu \nu} \sec \varphi_{\kappa} \\
& \times \cos \left\{(2 l-1) \varphi_{\kappa}\right\} \cos \left\{(2 m-1) \chi_{\mu^{\prime}}\right\} \cos \left((2 n-1) \phi_{\nu}\right\} .
\end{aligned}
$$

\section{Three-Dimensional Monatomic Lattice with Isotopic Impurities on one of the Boundaries}

First we consider a three-dimensional monatomic lattice with an isotopic impurity on one of the boundaries. In the same way as in $\$ 5$, we can extend the formulas in $\$ 6$ to include the effect of the impurity at a lattice point $\left(N_{1}, j, k\right)$.

Introducing, as before, the extra lattices, we write the equation of motion in vector-matrix forms :

$$
\begin{aligned}
& x(l+1, m, n)=T^{\prime} x(l, m, n), \quad\left\{\begin{array}{l}
l=N_{1}, \\
m=j, 2 N_{2}-j+1, \cdots, \\
n=k, 2 N_{3}-k+1, \cdots,
\end{array}\right. \\
& x(l+1, m, n)=T x(l, m, n), \quad \text { otherwise }, \\
& \left\{\begin{array}{l}
x(1, m, n)=\left(\begin{array}{l}
1 \\
1
\end{array}\right) u(1, m, n), \\
x\left(N_{1}+1, m, n\right)=\left(\begin{array}{l}
1 \\
1
\end{array}\right) u\left(N_{1}, m, n\right),
\end{array}\right.
\end{aligned}
$$

where

$$
T^{\prime}=T-\left(\begin{array}{ll}
\delta & 0 \\
0 & 0
\end{array}\right),
$$




$$
\begin{aligned}
& \delta=Q M \omega^{2} / K_{1}, \\
& Q=\left(M^{\prime}-M\right) / M, \\
& M \omega^{2}=4 K_{1} \sin ^{2} \theta_{\mu \nu}+4 K_{2} \sin ^{2} \chi_{i t}+4 K_{3} \sin ^{2} \phi_{\nu},
\end{aligned}
$$

and

$$
u(1, m, n)=\sum_{\mu=0}^{2 N_{z}-1} \sum_{\nu=0}^{2 N_{3}-1} c_{\mu \nu} Z_{\mu \nu}(m, n) .
$$

From Eqs. (7.1a) and (7.1b) we obtain

$$
x\left(N_{1}+1, m, n\right)=T^{\prime} T^{N_{1} \cdots 1} x(1, m, n), \quad\left\{\begin{array}{l}
m=j, 2 N_{2}-j+1, \cdots, \\
n=k, 2 N_{3}-k+1, \cdots,
\end{array}\right.
$$

and

$$
x\left(N_{1}+1, m, n\right)=T^{N_{1}} x(1, m, n), \quad \text { otherwise } .
$$

Combined with Eqs. $(7.1 \mathrm{c}$ ), we get

$$
\sum_{\mu=0}^{2 N_{2}-2} \sum_{\nu=0}^{2 N_{3}-1} c_{\mu \nu}\left(A_{\mu \nu}-\delta B_{\mu \nu}\right) Z_{\mu \nu}(m, n)=0, \quad\left\{\begin{array}{l}
m=j, \cdots \\
n=k, \cdots
\end{array}\right.
$$

and

$$
\sum_{\mu=1}^{2 N_{2}-1} \sum_{\nu=1}^{2 N_{3}-1} c_{\mu \nu} A_{\mu \nu} Z_{\mu \nu}(m, n)=0, \quad \text { otherwise }
$$

where

$$
A_{\mu \nu}=S_{\mu \nu}\left(N_{1}+1\right)-2 S_{\mu \nu}\left(N_{1}\right)+S_{\mu \nu}\left(N_{1}-1\right)
$$

and

$$
B_{\mu \nu}=S_{\mu \nu}\left(N_{1}\right)-S_{\mu \nu}\left(N_{1}-1\right) .
$$

By the use of the orthogonality relation (6.10), the equations (7.3) are reduced to

$$
\begin{aligned}
0= & c_{\mu^{\prime}} A_{\mu \nu}-\cos \left\{(2 j-1) \chi_{\mu}\right\} \cos \left\{(2 k-1) \phi_{\nu}\right\} \\
& \times \frac{\delta}{N_{2} N_{3}} \sum_{\mu^{\prime}=0}^{N_{2}-1} \sum_{\nu^{\prime}-0}^{N_{3}-1} c_{\mu^{\prime} \nu^{\prime}}\left(2-\delta_{\mu^{\prime} 0}\right)\left(2-\delta_{\nu^{\prime} 0}\right) \cos \left\{(2 j-1) \chi_{\mu^{\prime}}\right\} \cos \left\{(2 k-1) \phi_{\nu^{\prime}}\right\} \\
& \times B_{\mu^{\prime} \nu^{\prime}}
\end{aligned}
$$

The condition for the existence of a non-trivial solution is

$$
\begin{aligned}
D= & \prod_{(\mu, \nu)=(0,0)}^{\left(N_{2}-1, N_{3}-1\right)} A_{\mu \nu}-\frac{\delta}{N_{2} N_{3}} \sum_{\mu=0}^{N_{2}-1} \sum_{\nu=0}^{N_{3}-1}\left(2-\delta_{\mu 0}\right)\left(2-\delta_{\nu 0}\right) \cos ^{2}\left\{(2 j-1) \chi_{\mu}\right\} \\
& \times \cos ^{2}\left\{(2 k-1) \phi_{\nu}\right\} B_{\mu \nu} \prod_{\left(\mu^{\prime} \nu^{\prime}\right) \neq(\mu \nu)} A_{\mu^{\prime} \nu^{\prime}}=0
\end{aligned}
$$

From this we can find the perturbed eigenfrequencies which resemble in character to those in $\$ 5$. The unperturbed eigenfrequency corresponding to the 
unperturbed normal mode which has a node at the impurity site remains to be a perturbed eigenfrequency.

In order to get other eigenfrequencies which are affected by the presence of impurity, we rewrite Eq. (7.5) as follows:

$$
1-\frac{\delta}{N_{2} N_{3}} \sum_{\mu=0}^{2 N_{2}-12 N_{3}-1} \sum_{\nu=0}^{2} \cos ^{2}\left\{(2 j-1) \chi_{\mu}\right\} \cos ^{2}\left\{(2 k-1) \psi_{\nu}\right\} \frac{B_{\mu \nu}}{A_{\mu \nu}}=0 .
$$

This turns out to be

$$
\begin{gathered}
1+\frac{\delta}{2}\left\{1+\frac{1}{N_{2} N_{3}} \sum_{\mu=0}^{2 N_{p}-1} \sum_{\nu=0}^{N_{3}-1} \cos ^{2}\left\{(2 j-1) \chi_{\mu}\right\} \cos ^{2}\left\{(2 k-1) \psi_{\nu}\right\} \cot \theta_{\mu \nu}\right. \\
\left.\times \cot 2 N_{1} \theta_{\mu \nu}\right\}=0
\end{gathered}
$$

where we used the following relations:

$$
\begin{aligned}
& \frac{B_{\mu \nu}}{A_{\mu \nu}}=-\left(1+\cot \theta_{\mu \nu} \cot 2 N_{1} \theta_{\mu \nu}\right) / 2, \\
& \sum_{\mu=0}^{2 N_{2} \nu-1} \cos ^{2}\left\{(2 j-1) \chi_{\mu \prime}\right\}=N_{2},
\end{aligned}
$$

and

$$
\sum_{\nu=1}^{2 N_{3}-1} \cos ^{2}\left\{(2 k-1) \phi_{\nu}\right\}=N_{3} .
$$

From these results it is seen that there may be only one extra-band eigenfrequency in the case $Q<0$ which is given by

$$
1+\frac{Q M \omega^{2}}{2 K}\left\{1-\frac{1}{N_{2} N_{3}} \sum_{\mu=0}^{2 N_{z}-12 N_{s}-1} \sum_{\nu=0} \cos ^{2}\left\{(2 j-1) \chi_{\mu}\right\} \cos ^{2}\left\{(2 k-1) \phi_{\nu}\right\} \tanh \varepsilon_{\mu \nu}\right\}=0
$$

and

$$
\tanh \varepsilon_{\mu \nu}=\left(\frac{M \omega^{2}-4 K_{2} \sin ^{2} \chi_{\mu}-4 K_{3} \sin ^{2} \psi_{\nu}-4 K_{1}}{M \omega^{2}-4 K_{2} \sin ^{2} \chi_{\mu}-4 K_{3} \sin ^{2} \phi_{\nu}}\right)^{\frac{1}{2}}
$$

where we put

$$
\theta_{\mu \nu}=\pi / 2+i \varepsilon_{\mu \nu}
$$

and assumed that

$$
\operatorname{coth} 2 N_{1} \varepsilon_{\mu \nu} \cong 1, \quad \text { i.e., } \quad \varepsilon_{\mu \nu} \gg 1 / N_{1} .
$$

Substituting the value $M \omega^{2}=4 K_{1}+4 K_{2}+4 K_{3}$ into Eq. (7.7a), we obtain the critical value $Q_{0}$ below which the extra-band frequency appears: 


$$
\begin{gathered}
1-\frac{2 Q_{c}\left(K_{1}+K_{2}+K_{3}\right)}{K_{1}}\left\{1-\frac{1}{N_{2} N_{3}} \sum_{\mu=0}^{2 N_{2}-1} \sum_{\nu=0}^{2 N_{3}-1} \cos ^{2}\left\{(2 j-1) \chi_{\mu}\right\} \cos ^{2}\left\{(2 k-1) \phi_{\nu}\right\}\right. \\
\left.\times\left(\frac{K_{2} \cos ^{2} \chi_{\mu}+K_{3} \cos ^{2} \phi_{\nu}}{K_{1}+K_{2} \cos ^{2} \chi_{\mu}+K_{3} \cos ^{2} \phi_{\nu}}\right)^{\frac{1}{2}}\right\}=0
\end{gathered}
$$

Next we consider a three-dimensional lattice, one of the surfaces of which $\left(l=N_{1}\right)$ is entirely composed of impurities of the same kind. This is a straightforward generalization of the case in $\$ 5$ (Eqs. (5.24) $\sim(5.32)$ ).

From Eqs. (7.2a) and (7.3a), we get

$$
\sum_{\mu=0}^{2 N_{2}-1} \sum_{\nu=0}^{2 N_{3}-1} c_{\mu \nu}\left(A_{\mu \nu}-\delta B_{\mu \nu}\right) Z_{\mu \nu}(m, n)=0 \quad \text { for all }(m, n) .
$$

By the use of the orthogonality relation $(6.10)$ of the functions $Z_{\mu \nu}(m, n)$, we obtain the condition for Eq. (7.9) to have a non-trivial solution :

$$
A_{r \nu}-\delta B_{\mu \nu}=0, \quad\left\{\begin{array}{l}
\mu=0,1, \cdots, N_{2}-1, \\
\nu=0,1, \cdots, N_{3}-1 .
\end{array}\right.
$$

From this we find the relation between the value of $Q$ and the complex root $\theta_{\mu \nu}$ :

$$
Q=-\frac{1}{2} \frac{\exp \left(\varepsilon_{\mu \nu}\right) \cosh \varepsilon_{\mu \nu}}{\cosh ^{2} \varepsilon_{\mu \nu}+\left(K_{2} / K_{1}\right) \sin ^{2} \chi_{\mu \nu}+\left(K_{3} / K_{1}\right) \sin ^{2} \phi_{\nu}}
$$

where we put

$$
\theta_{t, \nu}=\pi / 2+i \varepsilon_{\mu \nu}\left(\varepsilon_{\mu \nu}>0\right)
$$

The critical value $Q_{\mu \nu}$ for the appearance of the complex root $\theta_{\mu \nu}$ is given by

$$
Q_{\mu \nu}=-\frac{1}{2} \frac{K_{1}}{K_{1}+K_{2} \sin ^{2} \chi_{\mu}+K_{3} \sin ^{2} \psi_{\nu}} .
$$

In the same way as in $(\$ 6)$, we get the normal mode corresponding to the root $\theta_{\mu \nu}$ of Eq. (7.10):

$$
\begin{aligned}
& u(l, m, n)=\left(2-\delta_{\mu 0}\right)\left(2-\delta_{\nu 0}\right) c_{\mu \nu} \sec \theta_{\mu \nu} \\
& \quad \times \cos \left\{(2 l-1) \theta_{\mu \nu}\right\} \cos \left\{(2 m-1) \chi_{\mu}\right\} \cos \left\{(2 n-1) \phi_{\nu}\right\} .
\end{aligned}
$$

Especially, for the complex root $\theta_{\mu \nu}$ it follows that

$$
u(l, m, n) \cong(-1)^{N_{1}-\imath} \exp \left\{-2\left(N_{1}-1\right) \varepsilon_{\mu \nu}\right\} u\left(N_{1}, m, n\right),
$$

where

$$
u\left(N_{1}, m, n\right) \sim \cos \left\{(2 m-1) \chi_{\mu}\right\} \cos \left\{(2 n-1) \phi_{\nu}\right\},
$$

and we assumed that $\varepsilon_{\mu \nu} \gg 1 / N_{1}$. 


\section{Lattices with an Isotopic Impurity Atom at its any Point}

So far we have considered the lattices with impurities at its extremity. The method of transfer-matrix is effective also for the lattices with an impurity at its any point. In this section, however, we show that we can deduce directly from the results obtained in the sections 5 and 7 the conclusions for such cases.

Case (A). If we put $N_{1}=1$ in $\$ 5$, the lattice is reduced to the onedimensional lattice with an impurity at the lattice point $(j)$.

From Eqs. $(5.6 \mathrm{~b}, \mathrm{c})$, it follows that

$$
A_{n}=-4 \sin ^{2} \theta_{n}=M\left(\omega_{0}^{2}(\mu)-\omega^{2}\right) / K_{1},
$$

and

$$
B_{u}=1,
$$

where we used

and

$$
M \omega^{2}=4 K_{1} \sin ^{2} \theta_{\mu}+4 K_{2} \sin ^{2} \chi_{\eta^{\prime \prime}},
$$

$$
M \omega_{0}^{2}(\mu)=4 K_{2} \sin ^{2} \chi_{n} .
$$

In order to get the eigenfrequencies affected by the presence of the impurity, we substitute Eqs. (8.1) into Eq. (5.9b):

$$
1+Q \omega^{2} \frac{1}{N_{2}} \sum_{n=0}^{2 N_{2}-1} \frac{\cos ^{2}\left\{(2 j-1) \chi_{n}\right\}}{\omega^{2}-\omega_{0}^{2}(\mu)}=0 .
$$

Case (B). If we put $N_{1}=1$ in $\S 7$, the lattice is reduced to the twodimensional lattice with an impurity at the lattice point $(j, k)$.

From Eqs. (7.3), it follows that

$$
A_{\mu \nu}=-4 \sin ^{2} \theta_{\mu \nu}=M\left(\omega_{0}^{2}(\mu, \nu)-\omega^{2}\right) / K_{1},
$$

and

$$
B_{p: \nu}=1,
$$

where we used

and

$$
M \omega^{2}=4 K_{1} \sin ^{2} \theta_{\mu \nu}+4 K_{2} \sin ^{2} \chi_{\mu}+4 K_{3} \sin ^{2} \phi_{\nu}
$$

$$
M \omega_{0}^{2}(\mu, \nu)=4 K_{2} \sin ^{2} \chi_{\mu}+4 K_{3} \sin ^{2} \psi_{\nu} .
$$

The equation (7.6a) turns out to be

$$
\begin{gathered}
1+Q \omega^{2} \frac{1}{N_{2} N_{3}} \sum_{\mu=0}^{2 N_{2}-12 N_{3}-1} \sum_{\nu=0}^{\cos ^{2}\left\{(2 j-1) \chi_{\mu}\right\} \cos ^{2}\left\{(2 k-1) \phi_{\nu}\right\}} \\
\omega^{2}-\omega_{0}^{2}(\mu, \nu) \\
-160-
\end{gathered}
$$


Case (C). The formulas obtained in Cases (A) and (B) are easily generalized to the three-dimensional lattice with an impurity at the lattice point $(i . j . k)$ :

$$
\begin{aligned}
1+Q \omega^{2} \frac{1}{N_{1} N_{2} N_{3}} \sum_{k=0}^{2 N_{1}-1} \sum_{\mu=0}^{2 N_{2}-1} \sum_{\nu=0}^{2 N_{3} \cdots-1} \frac{\cos ^{2}\left\{(2 i-1) \varphi_{k}\right\} \cos ^{2}\left\{(2 j-1) \chi_{\mu}\right\} \cos ^{2}\left\{(2 k-1) \phi_{\nu}\right\}}{\omega^{2}-\omega_{0}^{2}(\kappa, \mu, \nu)} \\
=0,
\end{aligned}
$$

where

$$
M \omega_{0}^{2}(\kappa, \mu, \nu)=4 K_{1} \sin ^{2} \varphi_{\kappa}+4 K_{2} \sin ^{2} \chi_{\mu}+4 K_{3} \sin ^{2} \psi_{\nu} .
$$

It. is readily seen that the above results are no other than the usual one obtained by GREEN's function method, in which the equation of motion is solved by expanding the displacements in eigenfunctions which satisfy the homogeneous equation of motion with the imposed boundary conditions.

\section{Acknowledgments}

The author would like to thank Professor T. Toy A for his interest in this work and his continual encouragement. $\mathrm{He}$ is particularly grateful to Dr. J. HoRI, who made several valuable comments.

\section{APPENDIX}

In this appendix we show that the difference operators $d^{2}(l)$ which are originally dependent on the positions in $l$-direction can be treated as if they did not depend on $l$ under suitable conditions.

From Eqs. (4.4) it follows that

$$
\begin{aligned}
x(l+1, m) & =T(l, m) x(l, m) \\
& =\left(\begin{array}{cr}
\gamma(l, m)-f \Delta^{2}(l) & -1 \\
1 & 0
\end{array}\right)\left(\begin{array}{l}
u(l, m) \\
u(l-1, m)
\end{array}\right) \\
& \left.=\left(\begin{array}{cr}
\gamma(l, m)-f \Delta^{2}(l) & -1 \\
1 & 0
\end{array}\right) T(l-1, m) x(l-1, m), \quad \text { (A. } 1 \mathrm{c}\right)
\end{aligned}
$$

and

$$
\gamma(l, m)=2-M(l, m) \omega^{2} / K_{1},
$$

$$
f=K_{2} / K_{1} \text {. }
$$

Using the definition of $A^{2}(l)$, Eq. $(4.4 \mathrm{c})$, we get

$$
\begin{gathered}
J^{2}(l)[T(l-1, m) x(l-1, m)]_{1}, \\
\quad=J^{2}(l) u(l, m)
\end{gathered}
$$




$$
\begin{aligned}
= & u(l, m-1)-2 u(l, m)+u(l, m-1) \\
= & {[T(l-1, m-1) x(l-1, m-1)-2 T(l-1, m) x(l-1, m)} \\
& \quad+T(l-1, m+1) x(l-1, m+1)]_{1},
\end{aligned}
$$

where the suffix indicate the component of vector.

If we assume that

$$
T(l-1, m-1)=T(l-1, m)=T(l-1, m+1),
$$

then the equation (A. 2 ) is reduced to

$$
J^{2}(l)[T(l-1, m) x(l-1, m)]_{t}=\left[T(l-1, m) d^{2}(l-1) x(l-1, m)\right]_{1}
$$

where we defined the new difference operator $d^{\prime 2}(l)$ by

$$
d^{\prime 2}(l) x(l, m)=x(l, m-1)-2 x(l, m)+x(l, m-1) .
$$

Since the operator $J^{2}(l-1)$ contained within $T(l-1, m)$ on the right-hand side of Eq. (A. 4 a) can be replaced by $d^{\prime 2}(l-1)$, it follows that

$$
\Delta^{2}(l)[T(l-1, m) x(l-1, m)]_{1}=\Delta^{\prime 2}(l-1)[T(l-1, m) x(l-1, m)]_{1}
$$

\section{(A. $4 \mathrm{c})$}

Combined with Eq. (A.1c), we conclude that the operators $\Delta^{2}(l)$ and $\Delta^{2}(l-1)$ which appear in $T(l, m)$ and $T(l-1, m)$ respectively can be replaced by $d^{\prime 2}(l-1)$ provided that the equation of motion satisfy the condition (A.3). Consequently, we can rewrite Eq. (A. $1 \mathrm{c}$ ) as follows:

$$
\begin{aligned}
x(l+1, m) & =T(l, m) T(l-1, m) x(l-1, m) \\
& =S\left(\begin{array}{l}
u(l-1, m) \\
u(l-2, m)
\end{array}\right) \\
& =\left(\begin{array}{ll}
S_{11} & S_{12} \\
S_{21} & S_{22}
\end{array}\right) T(l-2, m) x(l-2, m)
\end{aligned}
$$

where the matrix element $S_{11}$ is a polynomial of the second order with respect to the operator $J^{\prime 2}(l-1), S_{12}$ and $S_{21}$ are of the first order, and $S_{22}$ is a constant. In the same way as in Eq. (A. 2), we obtain

$$
\begin{aligned}
d^{\prime 2}(l-1)[ & T(l-2, m) x(l-2, m)]_{2} \\
= & J^{\prime 2}(l-1) u(l-2, m) \\
= & {[T(l-2, m-1) x(l-2, m-1)-2 T(l-2, m) x(l-2, m)} \\
& \quad \mathbf{- 1 6 2 -}
\end{aligned}
$$


Some Applications of the Method of Transfer-Matrix to the Vibration of Lattices

$$
\begin{aligned}
& +T(l-2, m-1) x(l-2, m-1)]_{2}, \\
& d^{\prime 2}(l-1)[T(l-2, m) x(l-2, m)]_{1} \\
& =[T(l-2, m-1) x(l-2, m-1)-2 T(l-2, m) x(l-2, m) \\
& +T(l-2, m+1) x(l-2, m+1)]_{1}
\end{aligned}
$$

and

$$
\begin{aligned}
d^{\prime 4}(l-1) & {[T(l-2, m) x(l-2, m)]_{1} } \\
= & J^{\prime 4}(l-1) u(l-1, m) \\
= & L\{u(l-1, m-2), u(l-1, m-1), \cdots, u(l-1, m+2)\} \\
=L & \left\{[T(l-2, m-2) x(l-2, m-2)]_{1}, \cdots\right. \\
& \left.T(l-2, m+2) x(l-2, m+2)]_{1}\right\}
\end{aligned}
$$

where the function $L$ is a suitable linear combination of arguments.

If we assume that

$$
T(l-2, m-2)=T(l-2, m-1)=\cdots=T(l-2, m+2),
$$

then it follows that

$$
d^{\prime 2}(l-1) x(l-1, m)=d^{\prime 2}(l-2) T(l-2, m) x(l-2, m),
$$

and

$$
d^{\prime 4}(l-1) x(l-1, m)=d^{\prime 4}(l-2) T(l-2, m) x(l-2, m),
$$

where in the right-hand side the operator $J^{2}(l-2)$ contained in $T(l-2, m)$ is replaced by the operator $d^{\prime 2}(l-2)$. Combined with Eq. (A.5), we conclude that the operator $d^{\prime 2}(l-1)$ contained in the matrix $S$ can be replaced by the operator $d^{\prime 2}(l-2)$ provided that the equation of motion satisfy the condition (A. 7).

From these results it is clear that the difference operator $J^{2}(l)$ at any lattice point $(l, m)$ can be replaced by the operator $d^{\prime 2}(1)$ provided that the same kind of conditions as Eqs. (A.3) and (A.7) are satisfied all over the "domain of influence" of the point $(l, m)$.

\section{References}

1) T. AsAhi, Prog. Theor. Phys. Suppl., 23, 59 (1962).

2) J. Hori and T. AsAiri, Prog. Theor, Phys., 17, 523 (1957).

3) H. Matsuda, Prog. Theor. Phys. Suppl., 23, 22 (1962). 